\title{
Registro de ocorrência do besouro-escorpião Onychocerus albitarsis Pascoe (Coleoptera: Cerambycidae) no estado de São Paulo, Brasil
}

\author{
Antonio Lucas Sforcin Amaral ${ }^{\circledR}$, Antonio Leão Castilho, Jefferson Luan Crispim Rodrigues ${ }^{1}$,
} Ana Luiza Borges de Sá ${ }^{2}$ \& Vidal Haddad Jr3

1. Universidade Estadual Paulista "Julio de Mesquita Filho" - Instituto de Biociências de Botucatu. 2. Sem vínculo instituicional. 3. Departamento de Radiologia e Radioterapia, Faculdade de Medicina de Botucatu, Universidade Estadual Paulista - UNESP.

\section{EntomoBrasilis 12 (1): 27-30 (2019)}

Resumo. Besouros são insetos pertencentes à ordem Coleoptera, e compõem o grupo de animais mais diverso do mundo. Esta ordem inclui a família Cerambycidae, composta pelos besouros serra-pau, com algumas espécies consideradas pragas para as monoculturas arbóreas. Nessa família é encontrado o besouro-escorpião Onychocerus albitarsis Pascoe, que possui um par de ferrões na extremidade das antenas, e que pode causar acidentes em humanos. A ocorrência desta espécie é pouco conhecida e registros no estado de São Paulo, Brasil, são pobremente documentados. Dois espécimes foram encontrados nesta região após causarem lesões em humanos, sendo coletados para identificação. Registramos a ocorrência de O. albitarsis no estado de São Paulo, e estas informações serão úteis para futuros estudos entomológicos, ecológicos e médicos sobre esse animal.

Palavras-chave: Acidentes; animais peçonhentos; besouro serra-pau; Boituva; Botucatu.

\section{Occurrence record of scorpion-beetle Onychocerus albitarsis Pascoe (Coleoptera: Cerambycidae) in São Paulo State, Brazil}

Abstract. Beetles are insects belonging to the order Coleoptera, being the most diverse group of animals in the world. This order includes the family Cerambycidae, composed by saw-wood beetles, with some species considered pests to tree monocultures. In this family is found the scorpion-beetle Onychocerus albitarsis Pascoe, which has a pair of stingers at the end of antennae, and it may cause injuries in humans. The occurrence of this species is few known, and records in São Paulo state, Brazil, are poorly documented. Two specimens were found in this region after causing injuries in humans, and collected for identification. This work records the occurrence of O. albitarsis in São Paulo State, Brazil, and this information will be useful for further entomological, ecological and medical studies on the animal.

Keywords: Boituva; Botucatu; injury; longhorn beetle; venomous animals.

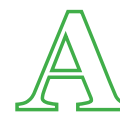

família Cerambycidae é uma das mais diversas da ordem Coleoptera, com cerca de 35.000 espécies, e abriga os besouros serra-pau, conhecidos pelo comportamento de cortar troncos de árvores (LAWRENCE 1982). Tal ação é observada em adultos, que com o aparelho bucal abrem buracos nos troncos e depositam seus ovos. Ao eclodirem, as larvas passam a se alimentar da madeira, e por conta disso, algumas espécies são consideradas pragas de culturas arbóreas, como de macieiras e salgueiros (TRIPLEHORN \& Johnson 2010). Algumas espécies da família, como Oncideres cingulata Say, são capazes de cortar galhos inteiros, que ao caírem e apodrecerem, se tornam um ambiente favorável ao desenvolvimento das larvas, que se alimentam da madeira em decomposição. Há espécies na família Cerambycidae que são capazes de gerar estalos intermitentes, friccionando a articulação do tórax com o abdome, fazendo um barulho que pode afugentar um predador (WEATHERSTON \& PERCY 1978; TRIPLEHORN \& JOHNSON 2010).
A subfamília Lamiinae é composta por algumas espécies consideradas pragas em monoculturas arbóreas. Nos gêneros Saperda e Oberea há espécies que podem causar prejuízos às culturas de macieiras, olmos, álamos e framboeseiras (TRIPLEHORN \& Johnson 2010). Nesta subfamília encontra-se a espécie Onychocerus albitarsis Pascoe, conhecida como besouroescorpião (Figura 1a). Possui cerca de $2 \mathrm{~cm}$ de comprimento, apresentando coloração predominante preta e branca com manchas amareladas. Possui antenas quase do comprimento do corpo, que atuam na quimiorrecepção e localização de alimento (PASCOE 1859; Allison et al., 2004). Podem ser encontradas em regiões de florestas neotropicais, como Mata Atlântica e Amazônica, havendo registros no Peru e Paraguai. No Brasil, foram descritos nos estados do Maranhão, Ceará, Bahia, Mato Grosso, Goiás, Espírito Santo, Rio de Janeiro e São Paulo (Julıo 2003; Berkov et al. 2008; Martins et al. 2009; BEZARK \& MonnÉ 2013; Tirant \& Limoges 2016). No entanto, a ocorrência no estado
Edited by:

William Costa Rodrigues

\section{Article History:}

Received: 29.x.2018

Accepted: 18.iii.2019
Corresponding author:

Antonio Lucas Sforcin Amaral

乃 antoniosfamaral@gmail.com

(3ttp://orcid.org/0000-0001-9903-1509
Funding agencies:

$\Delta$ Without funding declared 
de São Paulo é pouco conhecida, com registros em Amparo, Cubatão, Guarulhos, Peruíbe e Ribeirão Preto, sendo este último o mais recente, coletado em 1954 (Julio 2003). Registros indexados no estado após esse período não foram encontrados.

A espécie $O$. albitarsis possui um par de ferrões na ponta das antenas usado para defesa (Figura 1b), podendo causar acidentes em humanos (Smith 1884; Gahan 1899; Berkov et al. 2008). Tal característica faz com que suas antenas sejam comparadas à cauda dos escorpiões (Arachnida: Scorpiones), tornando-o conhecido como besouro-escorpião (Berkov et al. 2008). Porém, apesar da alegada semelhança, os órgãos inoculadores são análogos, uma vez que o ferrão de $O$. albitarsis está localizado na extremidade de um órgão sensorial do segmento cefálico, e o do escorpião é uma projeção da extremidade distal do segmento abdominal (metassoma) (PASCOE 1859; SMITH 1884; GAHAN 1899; Berkov et al. 2008; TRIPLEHORN \& JOHNSON 2010).

BERKov et al. (2008) descreveram que o ferrão do besouroescorpião possui dois poros de abertura, similar à estrutura de escoamento de veneno do escorpião Leiurus quinquestriatus Hemprich \& Ehrenberg (Scorpiones: Buthidae), o que sugere que O. albitarsis poderia injetar toxinas, caso as produza na região bulbosa do último segmento da antena. Adicionalmente, no mesmo estudo há o registro de secreções químicas filamentosas coaguladas nos poros do ferrão do besouro-escorpião, que poderiam ser as toxinas. No entanto, acidentes em humanos são pouco descritos, embora mencionados por GAHAN (1899) e descrito por Berkov et al. (2008). No presente trabalho são apresentados dois indivíduos de $O$. albitarsis, coletados a partir de acidentes envolvendo humanos, em duas cidades do estado de São Paulo que não possuíam registro de ocorrência do besouro.

\section{MATERIAL E MÉTODOS}

Os animais foram identificados após causarem lesões em dois humanos, que procuraram atendimento médico e levaram os besouros para identificação.

Identificação e deposição. Os espécimes foram identificados com o auxílio de chaves dicotômicas de identificação até o nível de subfamília, e a espécie através de caracteres-chave e ajuda de bibliografia especializada (PAscoe 1859; SMith 1884; GAHAN 1899; Julio 2003; Triplehorn \& JohnSON 2010). O material foi depositado na coleção de invertebrados do Departamento de
Zoologia do Instituto de Biociências de Botucatu da Universidade Estadual Paulista.

Comitê de Ética. A Faculdade de Medicina de Botucatu não exige aprovação prévia para publicação de dados anônimos referentes a seus pacientes.

\section{RESULTADOS}

\section{Espécime 1 (Figura 2a)}

Em fevereiro de 2018, no município de Boituva (Figura 3), no km 120 da Rodovia Presidente Castelo Branco SP-280 (23 ${ }^{\circ} 17^{\prime} 37.65$ " $\mathrm{S}, 47^{\circ} 43^{\prime} 14.13$ " O), um paciente de 28 anos, masculino, informou ter sido picado ao tocar um animal no posto de combustível na referida rodovia, enquanto abastecia o veículo. Logo a seguir, queixou-se de inflamação e dor no local da picada. O ambiente predominante no local é o rural, com fragmentos florestais de Cerrado e Mata Atlântica (floresta estacional semidecidual) (IBGE 1991).

\section{Espécime 2 (Figura 2b)}

Encontrado em abril de 2018, no município de Botucatu (Figura 3) dentro da Fazenda Lageado ( $22^{\circ} 50^{\prime} 27 \cdot 35$ ”S, $48^{\circ} 25^{\prime} 32.89$ ” O). A vítima de 29 anos, do sexo feminino, informou ter sentido a picada após esbarrar no animal em uma árvore, durante passeio no local que é composto por um fragmento de mata de transição entre Mata Atlântica (floresta estacional semidecidual) e Cerrado, inserido em uma zona rural (IBGE 1991). A lesão apresentou importante reação inflamatória no local, que persistiu por sete dias.

\section{DISCUSSÃO}

Estudos acerca da ocorrência do O. albitarsis são ausentes, especialmente no estado de São Paulo. Registros sobre a espécie indicam que poucos espécimes foram coletados na região, principalmente recentemente, e as circunstâncias em que foram encontrados, após acidente em humanos, indica a relevância de novos estudos sobre esta espécie. Além disso, esta espécie pertence a uma família importante para a agricultura, onde se encontram várias espécies consideradas pragas em monoculturas. A relação entre $O$. albitarsis e o cultivo de espécies arbóreas não está elucidada, carecendo de estudos mais aprofundados, bem como a sua importância médica. $\mathrm{O}$

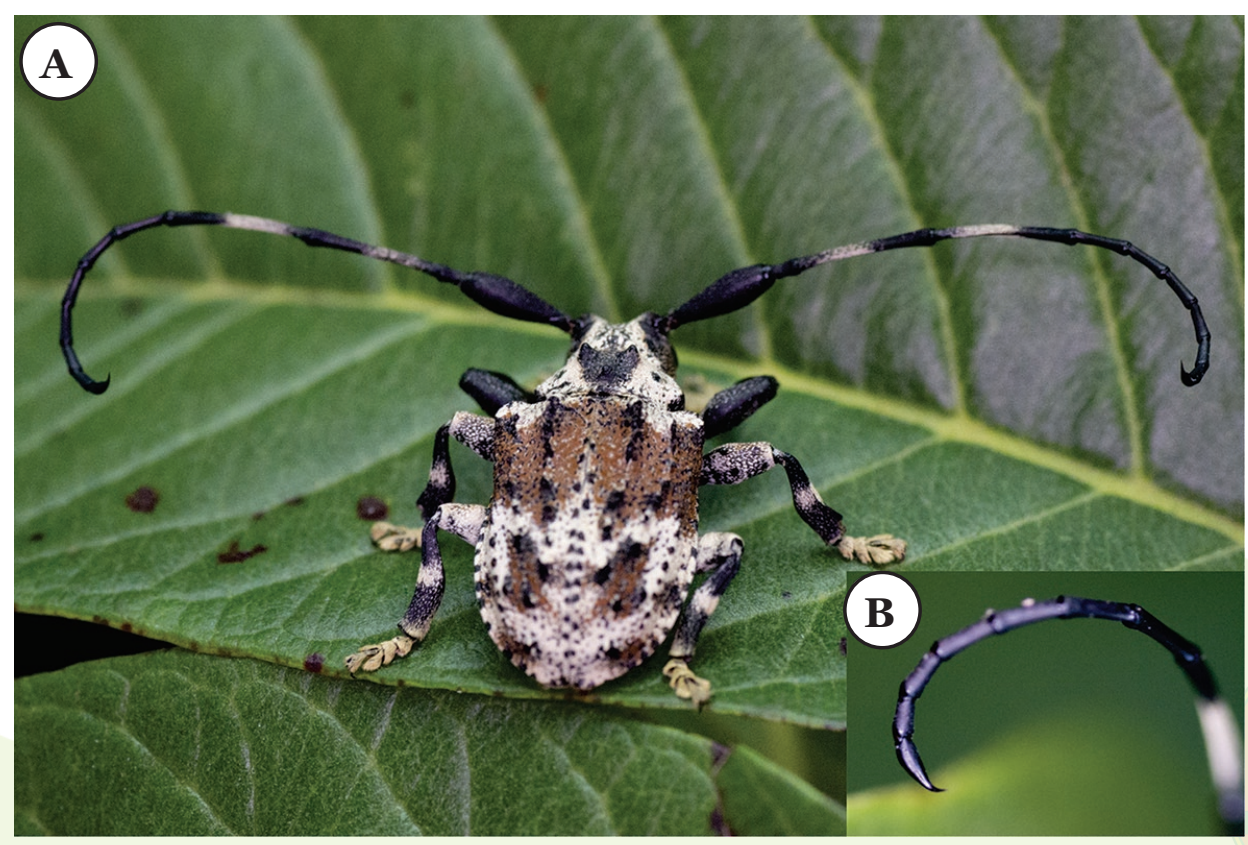

Figura 1. a) Onychocerus albitarsis vivo, no local de encontro relatado em Espécime 1. b) Detalhe do ferrão na extremidade distal da antena. Foto: A.L.S. Amaral. 


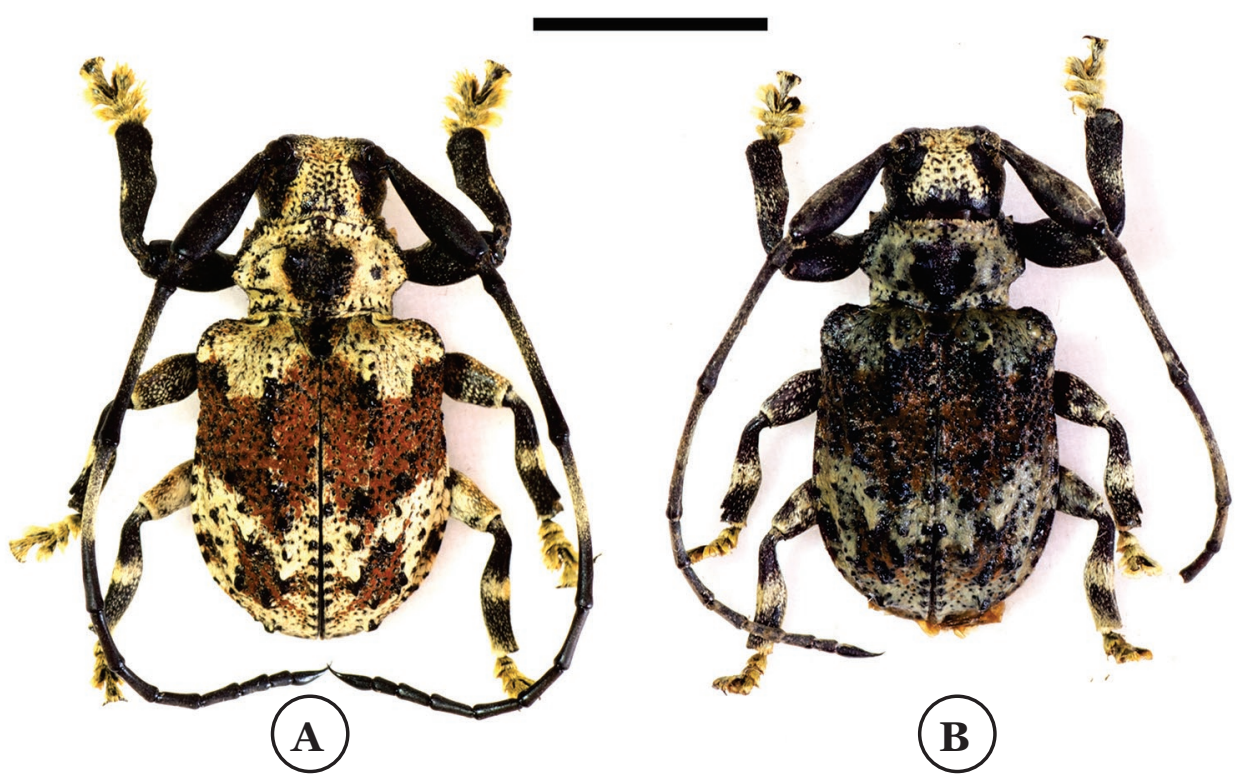

Figura 2. Indivíduos de $O$. albitarsis coletados após causarem as lesões em humanos. a) Espécime 1. b) Espécime 2, com alteração nas cores devido ao armazenamento até ser fixado na coleção de invertebrados do Departamento de Zoologia do Instituto de Biociências de Botucatu. Barra de escala: $1 \mathrm{~cm}$. Foto: A.L.S. Amaral.

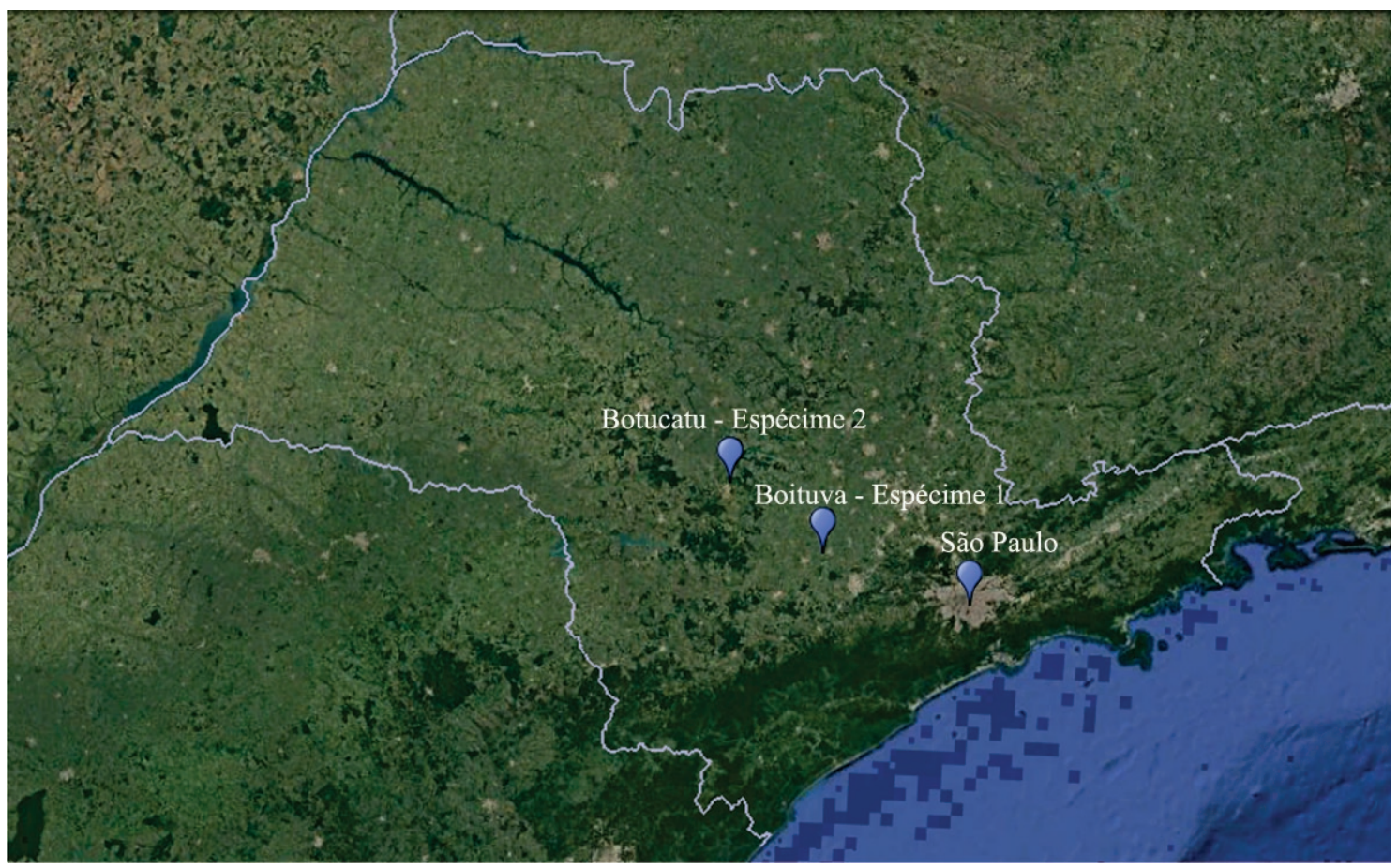

Figura 3. Imagem de satélite do estado de São Paulo, com destaque para a cidade de São Paulo (à direita) e as cidades onde foram encontrados os espécimes de O. albitarsis: espécime 1 em Boituva (abaixo) e espécime 2 em Botucatu (acima). Mapa: A.L.S. Amaral.

presente relato se faz importante, portanto, para futuros estudos entomológicos, ecológicos e médicos.

\section{AGRADECIMENTOS}

Agradecemos ao Dr. Carlos Eduardo de Alvarenga Julio, pelas informações complementares fornecidas sobre a ocorrência da espécie, e contribuição de material bibliográfico adicional. O primeiro autor possui bolsa de mestrado do Conselho Nacional do Desenvolvimento Científico e Tecnológico (CNPq).

\section{REFERÊNCIAS}

Allison, J.D., J.H. Borden \& S.J. Seybold, 2004. A review of the chemical ecology of the Cerambycidae (Coleoptera). Chemoecology, Birkhäuser-Verlag, Basel, 14: 123-150. DOI: https://doi.org/10.1007/s00049-004-0277-1.

Berkov, A., N. Rodríguez \& P. Centeno, 2008. Convergent evolution in the antennae of a cerambycid beetle, Onychocerus albitarsis, and the sting of a scorpion. Naturwissenschaften, 95: 257-261. DOI: https://doi.org/10.1007/s00114-0070316-1.

Bezark, L.G. \& M. Monné, 2013. Checklist of the Oxypeltidae, Vesperidae, Disteniidae and Cerambycidae, (Coleoptera) of the Western Hemisphere. 2013 ed., 484 p. Disponível em: $\quad$ https://plant.cdfa.ca.gov/byciddb/checklists/ WestHemiCerambycidae2013.pdf $>$

Gahan, C.J., 1899. Onychocerus albitarsis stinging with antennae. The Royal Enthomological Society, London, p.12-13.

IBGE - Instituto Brasileiro de Geografia e Estatística, 1991. Classificação da vegetação brasileira, adaptada a um sistema universal. Rio de Janeiro, IBGE, 123 p.

Julio, C.E.A., 2003. Revisão taxonômica e filogenia da tribo Anisocerini Thomson, 1860 (Coleoptera, Cerambycidae, Lamiinae). Tese (Doutorado em Biologia: Zoologia). Universidade Estadual Paulista, Instituto de Biociências de Botucatu. $254 \mathrm{f}$. 
Lawrence, J.F., 1982. Coleoptera. In: Parker, S.P. (ed.). Synopsis and Classification of Living Organisms, McGraw Hill, New York, 2: p.482-553.

Martins, U.R., M.H.M. Galileo \& F. Limeira-De-Oliveira, 2009. Cerambycidae (Coleoptera) do estado do Maranhão, Brasil. Papéis Avulsos de Zoologia, 49: 229-247. DOI: https://doi.org/10.1590/s0031-10492009003800001.

Pascoe, F.P., 1859. On New Genera and Species of Longicorn Coleoptera. The Royal Enthomological Society London, 10: 12-61.

Smith, H.H., 1884. Antennae of a beetle used as defensive weapons. The American Naturalist, 18: 727-728.
Tirant, S.L.E. \& R. Limoges, 2016. First record of Onychocerus albitarsis Pascoe, 1859 (Coleoptera: Cerambycidae: Lamiinae: Anisocerini) from Paraguay. Dugesiana, 23: 2.

Triplehorn, C.A. \& N.F. Johnson, 2010. Estudo dos insetos: Tradução da $7^{\mathrm{a}}$ edição norte-americana. 1 ed. São Paulo: Cengage Learning, 809 p.

Weatherston, J. \& J.E. Percy, 1978. Venoms of Coleoptera, p. 511-554. In: Bettini, S. (Org.), Arthropod Venoms. Springer, Berlin, Heidelberg.

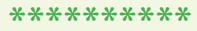

\section{Suggestion citation:}

Amaral, A.L.S., A.L. Castilho, J.L.C. Rodrigues, A.L.B. de Sá \& V. Haddad Jr, 2019. Registro de ocorrência do besouro-escorpião Onychocerus albitarsis Pascoe (Coleoptera: Cerambycidae) no estado de São Paulo, Brasil. EntomoBrasilis, 12 (1): 27-30.

Available on: doi:10.12741/ebrasilis.v12i1.822

\section{(c) (1) (8) (2)}
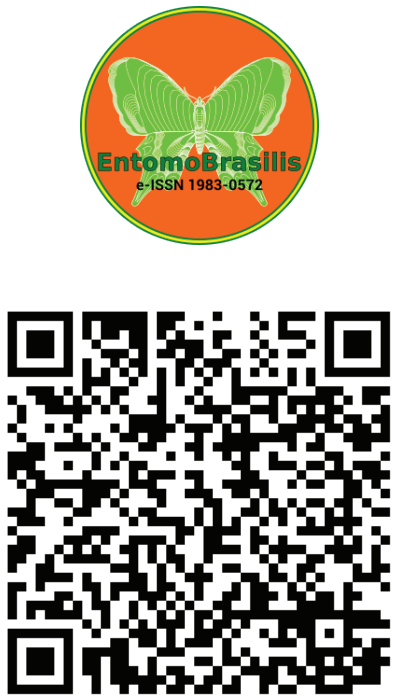